Further investigations revealed her ADAMTS13 (a metalloproteinase that cleaves von Willebrand factor) activity to be $<5$, confirming the diagnosis of TTP. ADAMTS13 activity of $68-163 \%$ is considered normal and activity of less than $10 \%$ is diagnostic of TTP. She is currently monitored as an outpatient and continues to have Rituximab for subclinical relapses.

Conclusion Bleeding of any form is an extremely rare and severe presentation of TTP. There are only a few case reports of TTP in children till date but there are no reported paediatric cases of symptomatic bleeding as initial presentation. Acquired TTP is mainly reported in adolescence. TTP is specifically related to severe ADAMTS13 deficiency which is also used to monitor disease activity.

\section{G185(P) INVESTIGATING CURRENT PRACTICES IN RENAL FUNCTION MEASUREMENT AND CARBOPLATIN DOSING IN CHILDREN WITH CANCER - A UK PERSPECTIVE}

${ }^{1} \mathrm{E}$ Roberts, ${ }^{1} \mathrm{~J}$ Mogg, ${ }^{2} \mathrm{M}$ Barnfield, ${ }^{1} \mathrm{G}$ Veal. 'Northern Institute for Cancer Research, Newcastle University, Newcastle upon Tyne, UK; ${ }^{2}$ Department of Medical Physics and Engineering, St James' University Hospital Leeds, Leeds, UK

\subsection{6/archdischild-2020-rcpch.156}

Introduction Renal function-based carboplatin dosing is a well accepted practice in paediatric oncology. However, the accuracy of this approach is only as precise as the method of kidney function measurement, most commonly involving determination of glomerular filtration rate (GFR). Recent work by the Children's Oncology Group has raised concerns over nuclear medicine-based methodologies used to calculate GFR across US clinical centres.

Aims The aims of this study were to evaluate current UK practices of GFR measurement, methods used to calculate carboplatin dosage and the utility of therapeutic drug monitoring in paediatric oncology services. This aims to provide an update on current practices across UK centres involved in the treatment of childhood cancer patients, and to measure change in practice since previous evaluation in 2008 .

Methods Twenty one primary paediatric oncology treatment centres in the UK provided information on carboplatin dosing practices in paediatric oncology patients through a questionnaire. Information obtained was compared to results previously published in 2008 following a similar study.

Results In relation to GFR measurement, the main changes observed were a shift towards a greater number of samples being taken following tracer administration and an increase in number of centres using the Brochner-Mortensen correction factor. In relation to the use of renal function assessment data to inform dosing, EDTA elimination half-life in conjunction with body weight was used to calculate carboplatin dose in 18/21 (86\%) centres, with uncorrected GFR and body weight utilised in 9/21 (43\%) centres. A total of 14/21 (67\%) centres utilise therapeutic drug monitoring approaches to carboplatin treatment in defined patient groups including neonates and infants.

Conclusion Results suggest that while GFR measurement across UK centres is relatively consistent, some uncertainties remain. In addition, for patient sub-populations where there are concerns over the potential for marked inter-patient variability in carboplatin exposures, adaptive dosing approaches are now well established.

\section{Paediatric educators' special interest group (PEdSIG)}

G186(P) ABSTRACT WITHDRAWN

\section{G187(P) TRAINING TOGETHER TO IMPROVE PAEDIATRIC PALLIATIVE CARE THROUGH INTERPROFESSIONAL SIMULATION}

${ }^{1} \mathrm{G}$ Balendran, ${ }^{2} \mathrm{~S}$ Vitello, ${ }^{1} \mathrm{~N}$ Sancroft, ${ }^{2} \mathrm{~F}$ Gilroy, ${ }^{1} \mathrm{~S}$ Kirk, ${ }^{1} \mathrm{~A}$ Kilonback. ${ }^{1}$ Paediatrics, Epsom Hospital, Epsom and St Helier NHS Trust, Epsom, UK; ${ }^{2}$ Simulation Centre, Epsom Hospital, Epsom and St Helier NHS Trust, Epsom, UK

\subsection{6/archdischild-2020-rcpch.157}

Aim Although there are rising numbers of children with lifelimiting diseases in the UK, there is a paucity of postgraduate paediatric palliative care training for both doctors and nurses. Current resources tend to focus on symptom management rather than the challenges of undertaking difficult conversations with patients and families. Interprofessional simulationbased education has been shown to improve both technical and human factor skills. We aimed to design an innovative interprofessional simulation-based course to improve confidence with paediatric palliative care.

Method The course was designed and delivered by an interprofessional faculty. Four scenarios were designed using a high-fidelity manikin and professional actress, focusing on key palliative care themes: advanced care planning, recognition of the dying patient, autonomy and care after death. The diamond debrief model was used to explore technical and human factor skills with a focus on real-life application. We also included a symptom management workshop.

Pre-and post-course questionnaires using Likert scales 0 to 100, (where $0=$ not at all and $100=$ totally agree) and free-text comments were completed by all participants to evaluate Kirkpatrick Levels one and two.

Results There were nine participants including paediatric consultants, paediatric registrars, senior paediatric nurses and oncology nurse specialists.

Feedback showed increases in average confidence levels. There was a 40-point (41 to 81) increase in confidence with having discussions surrounding dying, a 27-point (51 to 78) increase in confidence with DNAR discussions, and a 33point (46-79) and 30-point (51-81) increase in confidence with assessing and managing patients at the end of life respectively.

Highly positive feedback was received regarding the fidelity of the scenarios (91), the opportunity to practice clinical skills (83) and the usefulness of the feedback from the debrief sessions (93).

All of this was supported by qualitative data from free-text comments.

Conclusion This course is unique to our paediatric training region. Our feedback has shown that paediatric palliative care is an area in which many healthcare professionals lack confidence. We recommend interprofessional simulation training as an effective way of improving knowledge, confidence and non-technical skills essential for delivering paediatric palliative care to patients and their families. 